Additional, more sensitive, methods of monitoring drug treatment should expand the concept of "intolerable" side effects to include subtle psychological and behavioral effects. (Vining EPG et al. Pediatrics 1987; 80:165).

\title{
TERATOGENIC EFFECTS OF DIAZEPAM
}

Eight children exposed in utero to benzodiazepines, diazepam or oxazepam, and having dysmorphic characteristics resembling the fetal alcohol syndrome (FAS) are reported from the Department of Pediatrics, Gothenburg University, Sweden. The most common craniofacial abnormalities were slanted eyes, epicantic folds, short, uptilted nose, flat upper lip, and hypoplastic mandible. All were hypotonic from birth and had neonatal drug withdrawal symptoms of opisthotonus and convulsions. Low Apgar scores were recorded in 6 , mainly because of apnea, and 5 were resuscitated. Rooting and sucking reflexes were absent and feeding difficulties prominent. Gross motor disability in all children, 2 having spastic hemiparesis, was seen during early life, and fine motor incoordination and tremor were common in older children. One infant who died had neuronal migration defects and heterotopias. (Laegreid L et al. Teratogenic effects of benzodiazepine use during pregnancy. $\mathrm{J}$ Pediatr Jan $1989 ; \underline{114}: 126-31)$.

COMMENT. Alcohol ingestion in these mothers was excluded. The fetal diazepam syndrome (FDS) differed from the FAS in a greater focal involvement of cranial nerves, a sullen and expressionless facies, and more frequent occurrence of low Apgar scores, apneic spells, delayed motor development, and neonatal hypotonia. There have been numerous previous reports of the "floppy infant syndrome" in babies born to women treated with long-term diazepam during pregnancy. Bilateral opercular polymicrogyria and grey matter heterotopias found at autopsy of 2 cases of Foix-Chavany-Marie syndrome (faciopharyngealglossomasticatory diplegia) (Becker PS et al. Ann Neurol Jan 1989;25:90) might sometimes be explained by diazepam teratogenicity. This syndrome is rare and usually follows stroke and vascular infarction in adults. There are very few reports of the pathology in developmental varieties of the syndrome.

\section{MOVEMENT OF $\mathrm{X}$ CHROMOSOME IN EPILEPTIC CORTEX}

Chronic uncontrolled seizure activity was linked to specific positional changes of the $x$ chromosome in neurons of cortical seizure foci from both male and female patients at Yale University School of Medicine, New Haven, Connecticut. In normal female neurons, $X$ homologs were typically either on the nucleolus or on the nuclear membrane, whereas one or both $\mathrm{X}$ chromosome signals were moved interiorly in seizure foci of 2 representative female patients. There was a gradient of $x$ positional changes spreading from the seizure focus toward normal surrounding cortex. In seizure foci there was a decrease of $25 \%$ in membrane plus nucleolar signal neurons compared to more normal specimens, and a concomitant increase of $27 \%$ of 